\title{
Ein elektronisches Bundesgesetzblatt?
}

\author{
(Fortsetzung zu „Informatik und Recht” 1987, S. 43)
}

Merkwürdig genug: Auf dem Felde der CD-ROM-gestützten elektronischen Rechtsdokumentation hat man sich der für den Juristen unstreitig primären Informationsquelle, dem Gesetz nämlich, zuletzt zugewandt, erst deutlich nach Rechtsprechung und Literatur. Über die Gründe für die befremdliche Umkehrung der eigentlich notwendigen Reihenfolge nachzudenken ist der Mühe wert. Indessen ist mittlerweile dieser Zustand überwunden es gibt Bundesgesetzblatt und Bundesrecht auf CD-ROM. Es begann mit der jur-pc CD-ROM-Edition des Bundesgesetzblattes Teil I und II (jetzt in der melius-Reihe des Boorberg Verlages), die ab dem Stichtag 3. Oktober 1990 die Texte des Bundesgesetzblattes in elektronischer Form bietet. juris folgte mit der data disc „Bundesrecht” (vgl. hierzu jur-pc CD-ROM Digest 92, S. 112 ff.). (Von der Nomos-CD zum Thema Bundesrecht hat man hingegen seit der schon einige Zeit zurückliegenden Ankündigung mit Subskriptionsangebot nichts mehr gehört, sie kann also bei dem folgenden Vergleich außer Betracht bleiben.) Bei immerhin zwei Produkten besteht ausreichend Anlaß, den Blick nach vorn zu richten und die Konzeptionen zu befragen.

Eine methodische Kernfrage, zu der man Stellung beziehen muß, ist die, ob man eine konsolidierte elektronische Fassung des Bundesrechts vorlegen will oder ob man eine elektronische Version des Bundesgesetzblattes bietet, das bekanntlich in Gestalt von Änderungsgesetzen vièlfältige Anweisungen zur Änderung von Gesetzen beinhaltet und damit gerade nicht konsolidierte Texte zur Verfügung stellt. (In diesem Zusammenhang ist übrigens, was nicht allerorts bekannt ist, zu beachten, daß selbst die auf gesetzlichen Ermächtigungen beruhenden integralen Neubekanntmachungen von Gesetzestexten durch Bundesminister weder konstitutive noch deklaratorische Verbindlichkeit besitzen.)

In Anbetracht der geschilderten Alternative wählt juris mit Material des Bundesjustizministeriums den Weg der Konsolidierung, die melius-Edition bei Boorberg hingegen verfolgt das Ziel einer elektronischen Duplizierung des Original-Bundesgesetzblattes.

Unter Aspekten der Benutzerfreundlichkeit verdient scheinbar das Konzept der Konsolidierung (also der Einarbeitung von Änderungsanweisungen in den Gesetzestext) den Vorzug. Man verfügt auf diese Weise direkt über einen lesbaren Text. Für diesen Zustand bezahlt man allerdings in zweierlei Hinsicht einen Preis.

Die erste für eine Konsolidierung in Kauf zu nehmende Ungewißheit bringt das Handbuch zur juris data disc „Bundesrecht” offen und zutreffend auf folgenden Nenner:

"Die Texte der Vorschriften wurden gemäß den Grundsätzen des Gesetzes über die Sammlung des Bundesrechts vom 10. Juli 1958 (BGBl. 1 1958, Seite 437) vom Bundesminister der Justiz festgestellt. Die Feststellung bat weder konstitutiven noch deklaratorischen Rechtscharakter" (S. 5).

Der juristisch geschulte Leser wird sich fragen, welcher Verbindlichkeitsgrad jenseits des Konstitutiven und des Deklaratorischen noch übrigbleibt.

Der zweite mit der Konsolidierung einhergehende Informationsverlust besteht darin, daß man im konsolidierten Text nicht sofort sieht, an welcher Stelle eine Änderung vorgenommen wurde. Oft hängt aber die den Zweck einer Änderung ins Auge fassende Interpretation von einer Kenntnis der Textänderung ab, die - wie gesagt - im konsolidierten Text nicht direkt sichtbar ist. Auf diese Weise zieht der bei konsolidiertem Text bestehende Lesevorteil einen Verlust an Interpretationsmöglichkeiten nach sich.

Das alternative Konzept der unverändert-authentischen elektronischen Darbietung des BGBl.-Textes kann - insofern gleicht der Zustand dem bei der Konsolidierung - gleichfalls kein direktes amtliches Gütesiegel in Anspruch nehmen. Indessen verringert sich diese Problematik bei näherem Zusehen. Denn das gedruckte Bundesgesetzblatt verfügt über die beste denkbare Amtlichkeitsgarantie - die von Art. 82 Abs. 1 GG nämlich. Da nun die OCR-Technologie mittlerweile einen Stand erreicht hat, der (bei geeigneter Auswahl der

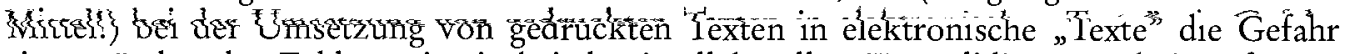
sinnverändernder Fehler, wie sie bei der intellektuellen Konsolidierungsarbeit auftreten können, ausschließt, besteht unter derartigen Rahmenbedingungen ein hohes Maß an praktischer Gewißheit, daß die elektronische Fassung der gedruckten entspricht.

Auch der zweite Nachteil einer Konsolidierung, der dargelegte Verlust der Interpretationsmöglichkeit „aus dem Änderungsbefehl”, ist bei einer unveränderten elektronischen Um-

- setzung des Bundesgesetzblattes vermieden, da der Änderungsbefehl original reproduziert wird.

Was bleibt als Fazit? Das muß der praktisch tätige Jurist selbst entscheiden. Die Entscheidung hängt in erster Linie davon ab, wie sehr die Sorge um den rechten Text des Gesetzes ihn umtreibt. Da Anstrengungen schmerzlich sind, ist eine Prognose über das Maß der vermutlichen diesbezüglichen Anstrengungen auch davon abhängig, welches Juristenbild man hat.

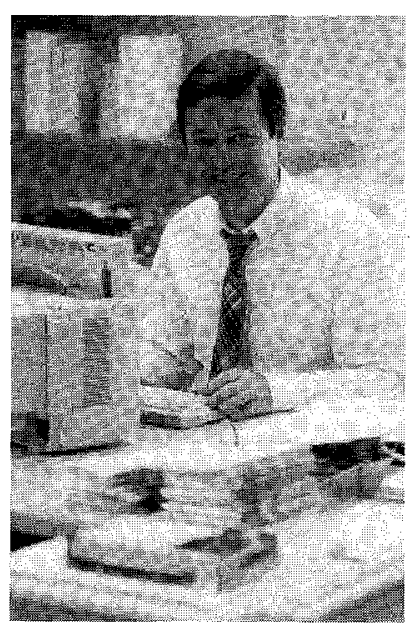

Gersweiler, 27. Oktober 1992

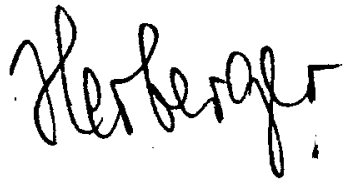

(Maximilian Herbergèr) 